\title{
Searching for the causal connection
}

\section{John Losee: Theories of Causality: from Antiquity to the Present. New Brunswick and London: Transaction Publishers, 2011, viii+210pp, \$59.95 HB}

\author{
Henk W. de Regt
}

Published online: 27 June 2012

(C) The Author(s) 2012. This article is published with open access at Springerlink.com

In contemporary debates about causality intuitions play an important role. A typical way of testing a proposed theory of causality is by examining to what extent it accommodates our intuitions about causal relations in concrete cases. We have strong intuitive ideas about what does and what does not qualify as a causal relation when we think, for example, about flagpoles and shadows, barometers and storms, or stones hitting windows. However, the fact that so many different, incompatible theories of causality have been proposed shows that it is difficult, if not impossible, to formulate a general theory that applies to all concrete cases, a single theory that satisfies all intuitions about causality.

One might of course ask why we should want a general theory of causality. One answer is that it can have a normative function, providing guiding principles for scientific practice. For example, a theory of causality is indispensable when designing experiments. If a specific theory that succeeds in this respect fails to comply with some of our intuitions, this should not count too heavily against it. Perhaps, a truly universal theory of causality is impossible because causality is not just one thing but rather a "cluster concept", a label for a number of partly overlapping ideas that do not share an essential core. John Losee's book, which summarizes 2,500 years of philosophical thinking about causality, concludes in such a spirit. Losee presents a "flowchart" that should help us in assessing causal relatedness for specific cases, distinguishing between three different domains for which different criteria apply.

The aim of Theories of Causality is "to present theories of causality within a historical survey that emphasizes the interrelationships between these theories and developments in science" (vii). As such, the book appears to be intended for classroom use, for example, in an undergraduate philosophy of science course.

H. W. de Regt ( $₫)$

Faculty of Philosophy, VU University Amsterdam, De Boelelaan 1105,

1081 HV Amsterdam, The Netherlands

e-mail: h.w.de.regt@vu.nl 
The material presented in the book does indeed provide a fairly complete overview of the most important philosophical accounts of causality since Aristotle. The main problem with this book, however, is that this historical overview is merely a long sequence of summaries of what particular philosophers have written about causality. What the book lacks is a coherent line of argument that relates the various theories and structures the debate about causality. This might have been achieved by classifying the theories in groups, or at least by clarifying their relations, similarities, and differences. For example, in Chapter 25 David Lewis's "counterfactual conditional view" is summarized. But the INUS theory of John Mackie and the manipulability account of James Woodward, which have been summarized in previous chapters, are also counterfactual theories. Losee does not even mention this and does not discuss the relation between Lewis's theory and these or other theories at all. This applies to almost all chapters that discuss individual theories: They are just summaries, without an attempt to relate them to the rest of the book. Chapters typically end quite abruptly, without concluding remarks or an evaluation of the theory discussed. In this respect, the textbook Causation and Explanation by Stathis Psillos (2002) provides much more insight, although it spends fewer pages on the topic of causality.

The book is divided into four parts. The first part reviews, in nine very short chapters, philosophical thinking about causality until 1900. First, there is a less than four-page chapter on Aristotle, then three pages on medieval science (with some tables that are not clearly explained), a chapter of less than three pages on Francis Bacon, and so on. A three-page chapter contains a very short discussion of Kant's account causality, with an unclear final paragraph. All in all, it would have been much better if these chapters had been integrated in one or two longer chapters, exposing the continuity of the debate.

Hume's influential "regularity theory" lies at the basis of most early-twentiethcentury discussions of causality and inspired the views of C. S. Peirce, Karl Pearson, Bertrand Russell, Norman Campbell, Moritz Schlick, and Henry Margenau, discussed in part II. Once again, it would have been preferable if the short chapters had been integrated into a cohesive story, showing the context of and relation between the various views. For example, Chapter 11 discusses Karl Pearson's view in less than two pages. The reader wants to know-but does not learn-who Karl Pearson was and why his view is important. In sum, the historical context that this book aspires to give is lacking. The chapter ends with a discussion of Pearson's instrumentalism, quoting a review of Pearson's book by Peirce, which does not mention causality at all. Instead of this, this reader would have preferred a comparison between Pearson's theory of causality and that of Peirce (which is the topic of Chapter 10).

Part III deals exclusively with causality in quantum mechanics. This is legitimate given that the book focuses on physical causation, for which quantum theory is highly relevant. Indeed, quantum theory was the first truly acausal theory in physics and this generated heated debates in the early twentieth century. Historian of science Paul Forman (1971) has suggested that the anti-intellectual climate in the Weimar Republic of the 1920s was responsible for an aversion against causality and induced physicists to develop acausal theories in atomic physics. Despite its historical orientation, Losee's book does not embed the theories in their historical context, however. Instead, part III 
begins with a relatively long exposition of quantum theory without explicit mention of causality, and subsequently presents a summary of Bohr's philosophy of complementarity including his analysis of causality. Chapter 15, entitled "Causality and the three levels of language in quantum mechanics," expands on the implications of complementarity for causality. Unfortunately, Losee does not explicitly state which "three levels of language" the title refers to. The final chapter of Part III deals with Philipp Frank's views on causality. Surprisingly, there is no explicit reference to quantum mechanics in this chapter. This is strange, not only because quantum mechanics is the central theme of Part III, but also because Frank actually wrote extensively on causality in relation to quantum theory. Thus, his important 1932 book The Law of Causality and Its Limits contains a separate chapter on this topic. However, Losee's account of Frank's views is based on passages from his 1957 introductory book Philosophy of Science - The Link between Science and Philosophy —and does not mention the 1932 monograph.

Part IV discusses theories that have been advanced as alternatives to the regularity view of causation. Among these are Curt Ducasse's theory of singular causation, the INUS theory of John Mackie, probabilistic theories of causality, Wesley Salmon's process theory, Phil Dowe's conserved quantity theory, David Fair's account of causality as energy or momentum transfer, theories of causes as powers (Rom Harré, Nancy Cartwright), manipulability theories (G. H. von Wright, Peter Menzies \& Huw Price, Judea Pearl, James Woodward), and finally, David Lewis's counterfactual view. Losee's discussions of these theories are more detailed than in Parts I and II, and they are generally clear and contain helpful examples. But these chapters also lack conclusions or comparative evaluations. For example, the chapter on manipulability theories discusses four different theories but makes no attempt at a comparative evaluation. Similarly, the chapter on causes as powers does not compare the views of Harré and Cartwright and neither does it relate these views to the material in the rest of the book. Finally, the chapter on Lewis ends with an odd paragraph on forces as causes that does not seem relevant to Lewis's theory at all.

The last three chapters of Part IV contain a rather unsystematic and uneven evaluation of many (but not all) of the previously discussed theories of causality. (It is strange that these chapters are in Part IV: They belong to the conclusions.) The evaluation results in a table listing the limitations of several criteria for causal status (175). To me, it seems that the manipulability theory comes out as the winner. As counterexamples to it, Losee only mentions large-scale physical processes, such as plate-tectonic effects, because classifying these as causal on the manipulability criterion would require "wild extrapolations from human practice" (175). But Woodward (2003, 127-33) defines "intervention"- the core notion of his manipulability theory - in a way that is completely independent of what humans can do. For some reason, however, Losee does not regard the manipulability theory as a serious contender. Instead, he investigates the prospects of a "disjunctive criterion" on which causality is either energy/momentum transfer or counterfactual dependence. He seems to favor the former criterion for "ordinary" physical causation and invokes the latter for cases of causal relatedness in which there is no energy or momentum transfer 
involved, like omission or prevention. This is a dubious choice, however: The energy/ momentum transfer criterion has been generally rejected as untenable, even in the domain of physics; see e.g. Dieks (1981) and Psillos (2002, 122). To be sure, Losee admits that energy/momentum transfer fails as a criterion for causality in the quantum domain and observes that a requirement of spatiotemporal continuity is problematic both in quantum and in classical physics (191-194). Surprisingly, however, he does not reject the energy/momentum transfer criterion. Because the disjunctive criterion appears problematic, Losee advances an alternative "flow chart for assessing causal relatedness" (199), which invokes the inferability criterion for quantum cases, the counterfactual dependency criterion for cases of omission, prevention or disconnection, and the energy/momentum transfer criterion for the remaining cases. It remains unclear why, given the problems with the latter criterion, Losee clings to it and does not include the much more promising manipulability criterion in his flowchart. Moreover, invoking inferability (which Losee strongly criticized on pp. 173-174) for quantum cases is an unacceptable ad hoc move. In sum, the final proposal appears arbitrary and unconvincing.

Theories of Causality could have been a useful introductory textbook. But both the author and the publisher should have spent much more time and effort on it. In its present form, it looks like a first draft of a manuscript or a course syllabus. While the book contains useful material, this should have been much better organized, to achieve a coherent account of the debate on causality instead of a long series of summaries. Moreover, these summaries are often unsystematic and uneven, and Losee's concluding assessment of them is unconvincing. Finally, the book contains many small mistakes and is sloppily edited. For example, in the formula on p. 64, the symbols are not explained and the minus sign should be a plus sign; years of birth and death are provided for some of historical figures but not for all; Helen Beebee's name is misspelled as "Beebe" (166-167); and bibliographical data in the endnotes are often incomplete (e.g., on p. 167).

A final remark: Losee writes that he focuses on physical causation, not on mental causation (viii). But this distinction does not exhaust possible types of causality: The social, human, and engineering sciences are full of causal reasoning that does not fit either of these categories. Moreover, several examples in the book are not about physical causation, for example, those involving the notions of responsibility and guilt (on p. 137 and pp. 185-86).

Open Access This article is distributed under the terms of the Creative Commons Attribution License which permits any use, distribution, and reproduction in any medium, provided the original author(s) and the source are credited.

\section{References}

Dieks, Dennis. 1981. A note on causation and the flow of energy. Erkenntnis 16: 103-108.

Forman, Paul. 1971. Weimar culture, causality, and quantum theory, 1918-1927. Historical Studies in the Physical Sciences 3: 1-115.

Psillos, Stathis. 2002. Causation and Explanation. Chesham: Acumen Publishers.

Woodward, James. 2003. Making Things Happen: A Theory of Causal Explanation. Oxford: Oxford University Press. 